\title{
N-Acetylcysteine Potentiates Inhibition of Platelet Aggregation by Nitroglycerin
}

\author{
Joseph Loscalzo \\ Division of Vascular Medicine and Atherosclerosis, Division of Cardiology, and Hemostasis Unit, Department of Medicine, \\ Brigham and Women's Hospital, and Harvard Medical School, Boston, Massachusetts 02115
}

\begin{abstract}
Platelet aggregation is currently felt to play an important role in the pathogenesis of ischemic vascular disorders. The smooth muscle relaxant, nitroglycerin, has been shown to inhibit platelet aggregation in vitro, but at concentrations that were felt to be unattainable in vivo. Because the in vivo action of nitroglycerin on smooth muscle cells has been shown to depend on the presence of reduced cytosolic sulfhydryl groups, the inhibitory effect of nitroglycerin on platelet aggregation was examined in the presence of the reduced thiol, $\boldsymbol{N}$-acetylcysteine. Millimolar concentrations of $\boldsymbol{N}$-acetylcysteine potentiated markedly the inhibitory effect of nitroglycerin on platelet aggregation induced by ADP, epinephrine, collagen, and arachidonate, decreasing the $50 \%$ inhibitory concentration (IC 5 ) $\sim 50$-fold for each of these agents. Other guanylate cyclase activators inhibited ADP-induced aggregation similarly and this inhibition was likewise potentiated by $\boldsymbol{N}$-acetylcysteine. Platelet guanosine 3',5'-cyclic monophosphate content increased fivefold in the presence of nitroglycerin and $\mathbf{N}$-acetylcysteine 2 min before maximal inhibition of ADP-induced aggregation was achieved, while simultaneously measured cyclic AMP did not change relative to base-line levels. In the absence of $\boldsymbol{N}$ acetylcysteine, nitroglycerin induced a marked decrease in platelet-reduced glutathione content as $S$-nitroso-thiol adducts were produced. The synthetic $S$-nitroso-thiol, $S$-nitroso- $N$ acetylcysteine, markedly inhibited platelet aggregation with an $\mathrm{IC}_{\text {so }}$ of $6 \mathrm{nM}$. These data show that $\boldsymbol{N}$-acetylcysteine markedly potentiates the inhibition of platelet aggregation by nitroglycerin and likely does so by inducing the formation of an $S$-nitrosothiol adduct(s), which activate guanylate cyclase.
\end{abstract}

\section{Introduction}

The importance of platelets in the pathogenesis of myocardial ischemia and infarction has recently been recognized. Circulating platelet aggregates are more prevalent in patients with sudden coronary death than in patients who die of other causes $(1,2)$. Vasoactive substances released by platelets at sites of endovascular injury, such as thromboxane $A_{2}$ and lipoxygenase products, produce coronary vasospasm (3-5). Furthermore, upon platelet activation, the platelet membrane itself serves as a catalytic surface on which coagulation factors interact in the formation of fibrin (6), the presence of which further limits vessel patency.

Address reprint requests to Dr. Loscalzo, Brigham and Women's Hospital, 75 Francis St., Boston, MA 02115.

Received for publication 11 February 1985.

J. Clin. Invest.

(c) The American Society for Clinical Investigation, Inc.

0021-9738/85/08/0703/06 $\$ 1.00$

Volume 76, August 1985, 703-708
Organic nitrate vasodilators, particularly nitroglycerin, have been used for many years in the treatment of ischemic heart disease (7). The mechanism of action of these agents remains incompletely defined $(8,9)$, but very likely involves direct smooth muscle relaxation in conductive (coronary) arteries $(10,11)$. Given the role of the platelet in the pathophysiology of coronary ischemia, several groups have investigated the effect of organic nitrate vasodilators on in vitro platelet function (12-14). Agonist-induced aggregation was uniformly inhibited by nitrates in these early studies, but the concentration of nitrates required was quite high and not achievable pharmacologically, thus calling into question the therapeutic relevance of this observation.

The work of Needleman et al. (15) demonstrated that the vasodilator action of nitroglycerin depends critically on the availability of certain essential sulfhydryl groups in vascular smooth muscle cells. These in vitro observations were extended in vivo by Horowitz et al. (16), who showed that pretreating patients with the sulfhydryl agent, $N$-acetylcysteine, reduced the amount of nitroglycerin required to achieve a targeted reduction in mean arterial and mean pulmonary capillary wedge pressures.

Because of the importance of platelets in the pathogenesis of myocardial ischemia, the ability of organic nitrates to inhibit platelet function in vitro (albeit at excessive concentrations), and the potentiating effect of sulfhydryl agents on nitroglycerin action in smooth muscle cells, we investigated the effect of $\mathrm{N}$-acetylcysteine on the inhibition of platelet aggregation by nitroglycerin and other organic nitrates. The data presented show the following: (a) that $N$-acetylcysteine markedly potentiates the inhibitory action of nitroglycerin on platelet aggregation in vitro to a variety of agonists; $(b)$ that nitroglycerin (as other nitrate vasodilators) inhibit platelet aggregation by increasing intracellular cyclic GMP (cGMP) and $N$-acetylcysteine markedly potentiates this effect; $(c)$ that nitroglycerin inhibits platelet aggregation through the formation of $S$-nitrosothiols, these latter compounds being both potent activators of guanylate cyclase and extremely potent inhibitors of platelet aggregation; and $(d)$ that pharmacologically achievable concentrations of nitroglycerin in vivo may act to inhibit platelet aggregation, provided that there are sufficient reduced sulfhydryl groups present in the platelet, and that, therefore, an important therapeutic action of nitroglycerin may well be inhibition of platelet activation.

\section{Methods}

Materials. Epinephrine, ADP, sodium azide, and 5,5'-dithiobis(2nitrobenzoic acid) (DTNB) ${ }^{1}$ were purchased from Sigma Chemical Co. (St. Louis, MO). Calf skin collagen was obtained from Worthington Biochemical (Freehold, NJ). Sodium arachidonate was purchased from

1. Abbreviations used in this paper: DTNB, 5,5'-dithiobis(2-nitrobenzoic acid); $I_{\text {so }}$, inhibitory concentration 50\%; PRP, platelet-rich plasma. 
NuCheck-Prep (Elysian, MN). Metaphosphoric acid, sulfanilamide, and $N$-(1-naphthyl)ethylenediamine dihydrochloride were purchased from Aldrich Chemical Co. (Milwaukee, WI). Nitroglycerin was obtained from Marion Laboratories, Inc. (Kansas City, MO). Sodium nitroprusside was purchased from Abbott Laboratories (Chicago, IL). N-Acetylcysteine was purchased from Mead Johnson and Co. (Evansville, IL) and from Duncan, Flockhart, and Co., (London, England). Reduced glutathione (GSH) was purchased from Calbiochem (LaJolla, CA). Radioimmunoassay kits for the determination of CGMP and cyclic AMP (cAMP) were purchased from New England Nuclear (Boston, MA). All other materials were reagent grade or better. Deionized water was used throughout.

Platelets. Venous blood was obtained within $1 \mathrm{~h}$ of use from volunteers who had not ingested acetylsalicylic acid for at least $10 \mathrm{~d}$ and was anticoagulated with $13 \mathrm{mM}$ sodium citrate. The platelet-rich plasma (PRP) was prepared by centrifugation at $160 \mathrm{~g}$ for $10 \mathrm{~min}$. Platelet counts were determined with a Coulter counter (model F; Coulter Electronics, Inc., Hialeah, FL).

Platelet aggregation. Platelet aggregation was monitored using a standard nephelometric technique (17) in which 0.4-ml aliquots of PRP were incubated at $37^{\circ} \mathrm{C}$ and stirred at $900 \mathrm{rpm}$ in a Payton dualchannel aggregometer (Payton Associates, Inc., Buffalo, NY). Aggregation was induced by addition of $11 \mu \mathrm{M}$ ADP, $12.5 \mu \mathrm{M}$ epinephrine, $0.12 \mathrm{mg} / \mathrm{ml}$ calf skin collagen, or $0.44 \mathrm{mM}$ arachidonate and changes in light transmittance recorded using an Omniscribe recorder (Houston Instruments, Austin, TX). PRP was preincubated at $37^{\circ} \mathrm{C}$ for $4 \mathrm{~min}$ with $N$-acetylcysteine or with nitroglycerin, or first with $N$-acetylcysteine and then nitroglycerin, and sodium nitroprusside, sodium azide, sodium nitrite, or $S$-nitroso- $N$-acetylcysteine for $1 \mathrm{~min}$ before addition of agonist. Aggregation was quantitated by measuring either the extent of change of light transmittance or the maximal rate of change of light transmittance; the extent of change in transmittance was used in experiments comparing effects of nitroglycerin or other nitrates on aggregation induced by different agonists, while the rate of change in transmittance was used in experiments addressing the time course of inhibition of aggregation.

Cyclic nucleotide assays. Measurements of cGMP and cAMP were performed by radioimmunoassay. After incubating PRP at $37^{\circ} \mathrm{C}$ with nitroglycerin and/or $N$-acetylcysteine, the platelets were processed as described previously (14) and radioimmunoassays for cGMP and cAMP performed. Acetylation of samples with acetic anhydride was used to increase the sensitivity of the assays.

Platelet glutathione measurements. GSH was measured in platelets by the method of Beutler et al. (18), with slight modifications. At various times after incubation with nitroglycerin, PRP was treated with $1.67 \%$ glacial metaphosphoric acid, $0.02 \%$ disodium (ethylenedinitrilo)tetraacetate, and $3 \% \mathrm{NaCl}$. The mixture was centrifuged at $8,700 \mathrm{~g}$ for $4 \mathrm{~min}$ at $4^{\circ} \mathrm{C}$ and the supernatant neutralized with $1.0 \mathrm{M}$ $\mathrm{Na}_{2} \mathrm{HPO}_{4}$. DTNB was used to detect free sulfhydryl groups spectrophotometrically $(19,20)$, using a molar extinction coefficient of $14,200 \mathrm{M}^{-1} \mathrm{~cm}^{-1}$ for the nitrothiophenolate ion (21).
Nitrite determination. Free nitrite was assayed in the supernatant of trichloroacetic acid extracts of platelets incubated with nitroglycerin with or without $\mathrm{N}$-acetylcysteine by the method of Snell and Snell (22), which involved diazotization of sulfanilic acid and subsequent coupling with the chromophore $N-(1$-naphthyl)ethylenediamine. $S$ Nitroso-thiols were detected by assaying free nitrite by diazotization of sulfanilic acid in the presence and absence of $0.15 \% \mathrm{HgCl}_{2}$, the latter reagent catalyzing the hydrolysis of $S$-nitroso bonds (23).

Preparation of S-nitroso- $\mathrm{N}$-acetylcysteine. S-Nitroso- $\mathrm{N}$-acetylcysteine was prepared at $25^{\circ} \mathrm{C}$ by reacting equimolar concentrations of $\mathrm{N}$ acetylcysteine with $\mathrm{NaNO}_{2}$ at acidic $\mathrm{pH}(24,25)$. Solutions turned from clear to rose-colored upon completion of the reaction. The $S$ nitroso-thiol was identified by visible absorption spectroscopy, having an absorption maximum of $550 \mathrm{~nm}$. The completeness of the reaction was verified by measuring free nitrite in the presence and absence of $0.15 \% \mathrm{HgCl}_{2}$, as described in the above paragraph, and by measuring reduced sulfhydryl groups using DTNB. Due to the instability of the $S$-nitroso-derivatives, $S$-nitroso- $N$-acetylcysteine was prepared within 1 $h$ of use, kept in acidic solution at $4^{\circ} \mathrm{C}$, and diluted as necessary into aqueous buffer immediately before addition to assay systems.

\section{Results}

Inhibition of platelet aggregation by nitroglycerin. Nitroglycerin inhibited platelet aggregation induced by $11 \mu \mathrm{M}$ ADP in a typical dose-response fashion (Fig. 1). The $\mathrm{IC}_{50}$ for this inhibition was $42 \mu \mathrm{M}$. Other agonists, including $12.5 \mu \mathrm{M}$ epinephrine, $0.12 \mathrm{mg} / \mathrm{ml}$ calf skin collagen, and $0.44 \mathrm{mM}$ arachidonate, were also inhibited with similar IC $_{50}$ s (Table I). For each agonist, the extent of aggregation at any concentration of nitroglycerin was normalized to that in the absence of nitroglycerin and $N$-acetylcysteine.

Potentiation of the inhibitory effect of nitroglycerin by $\mathrm{N}$ acetylcysteine. $N$-Acetylcysteine at $5.5 \mathrm{mM}$ added to PRP 4 $\mathrm{min}$ before the addition of nitroglycerin (and $5 \mathrm{~min}$ before the addition of agonist) markedly potentiated the inhibitory action of nitroglycerin for each agonist tested (Fig. 1 and Table I). The $\mathrm{IC}_{50}$ shifted from $\sim 42$ to $0.71 \mu \mathrm{M}$ for ADP with similar shifts noted for collagen, epinephrine, and arachidonate (Table I). At the concentration used in these experiments, $\mathrm{N}$-acetylcysteine alone did not inhibit platelet aggregation; however, at higher concentrations, inhibition of aggregation by $\mathrm{N}$-acetylcysteine alone was apparent. Lower concentrations of $\mathrm{N}$ acetylcysteine also potentiated inhibition by nitroglycerin, the effect decreasing with decreasing concentrations of $\mathrm{N}$-acetylcysteine.

Inhibitory effect of other guanylate cyclase activators on

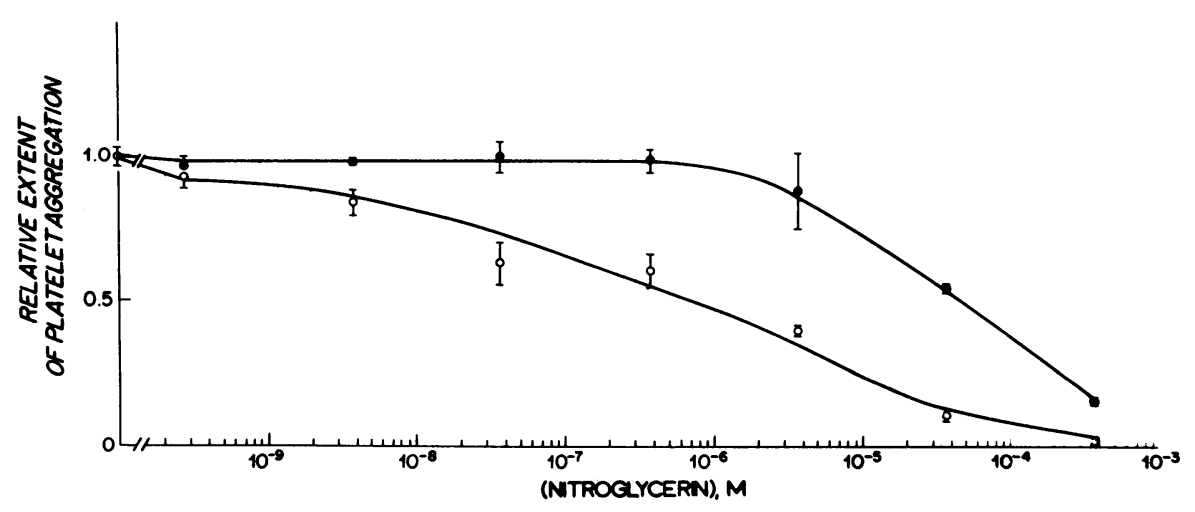

Figure 1. Potentiation of inhibition by nitroglycerin of ADP-induced platelet aggregation with $\mathrm{N}$-acetylcysteine. Platelets in PRP were incubated at $37^{\circ} \mathrm{C}$ with (o) or without (•) $5.5 \mathrm{mM} N$-acetylcysteine for 4 min, then with various concentrations of nitroglycerin for 1 additional min, after which $11 \mu \mathrm{M}$ ADP was added to initiate aggregation. Values on the ordinate reflect extent of aggregation relative to that in the absence of nitroglycerin and $N$-acetylcysteine (corresponding to $85 \%$ maximal light transmittance). Each point represents the mean \pm SEM of three experiments done in duplicate. 
Table I. Inhibition of Platelet Aggregation by Nitroglycerin and Potentiation of Inhibition by $\mathrm{N}$-Acetylcysteine*

\begin{tabular}{lll}
\hline & \multicolumn{2}{l}{$\mathrm{IC}_{s 0}$} \\
\cline { 2 - 3 } Agonist & $-N$-Acetylcysteine & $+N$-Acetylcysteine \\
\hline & $\mu M$ & $\mu M$ \\
ADP $(11 \mu \mathrm{M})$ & $42 \pm 2$ & $0.71 \pm 0.10$ \\
Epinephrine $(12.5 \mu \mathrm{M})$ & $34 \pm 4$ & $0.20 \pm 0.08$ \\
Collagen $(0.12 \mathrm{mg} / \mathrm{ml})$ & $36 \pm 4$ & $0.60 \pm 0.15$ \\
Arachidonate $(0.44 \mathrm{mM})$ & $45 \pm 6$ & $1.00 \pm 0.10$
\end{tabular}

* Aggregation of platelets in PRP was induced by addition of particular agonists after incubating platelets at $37^{\circ} \mathrm{C}$ with various concentrations of nitroglycerin $\pm 5.5 \mathrm{mM} \mathrm{N}$-acetylcysteine for $5 \mathrm{~min}$. $\mathrm{IC}_{50 \mathrm{~S}}$ each represent the mean \pm SEM for three experiments done in duplicate. For further details, see Methods.

ADP-induced platelet aggregation and potentiation by $\mathrm{N}$-acetylcysteine. Nitroglycerin is a potent activator of guanylate cyclase (26) and, given that this activation requires the presence of reduced thiol groups (26), other guanylate cyclase activators were tested for their ability to inhibit ADP-induced platelet aggregation in the presence and absence of $\mathrm{N}$-acetylcysteine. In Table II are listed the $\mathrm{IC}_{50}$ s for sodium acide, sodium nitroprusside, sodium nitrate, and nitroglycerin in the presence and absence of $5.5 \mathrm{mM} \mathrm{N}$-acetylcysteine. These data show that while the $\mathrm{IC}_{50} \mathrm{~S}$ ranged widely $(1.3 \mu \mathrm{M}$ for sodium azide to $59,000 \mu \mathrm{M}$ for sodium nitrite), in each case $N$-acetylcysteine potentiated the inhibition of ADP-induced aggregation, reducing the $\mathrm{IC}_{50}$, on average, 50 -fold (ranging from 22- to 87-fold for nitroprusside to nitrite, respectively).

Increase in platelet cGMP by nitroglycerin and potentiation by $\mathrm{N}$-acetylcysteine. Mellion et al. (27) showed that sodium nitroprusside and nitric oxide inhibited ADP-induced platelet aggregation and that this inhibition was preceded by an early, large, and partly transient increase in intracellular cGMP. For this reason, the effect of nitroglycerin on platelet cGMP was assessed and the influence of $\mathrm{N}$-acetylcysteine on this effect examined. Total platelet cGMP was measured by radioimmunoassay in quiescent platelets and platelets incubated with nitroglycerin with and without $\mathrm{N}$-acetylcysteine. Platelets in

Table II. Inhibition of Platelet Aggregation by Guanylate Cyclase Activators and Potentiation of Inhibition by N-Acetylcysteine*

\begin{tabular}{lcc}
\hline & \multicolumn{1}{l}{$\mathrm{IC}_{90}$} & \\
\cline { 2 - 3 } Activator & $-N$-Acetylcysteine & $+N$-Acetylcysteine \\
\hline & $\mu M$ & $\mu M$ \\
Sodium azide & 1.3 & 0.026 \\
Nitroglycerin & 42 & 0.80 \\
Sodium nitroprusside & 10 & 0.46 \\
Sodium nitrite & 59,000 & 680
\end{tabular}

* Aggregation of platelets in PRP was induced by addition of $11 \mu \mathrm{M}$ ADP after incubating platelets at $37^{\circ} \mathrm{C}$ with various concentrations of azide, nitroglycerin, nitroprusside, or nitrite $\pm 5.5 \mathrm{mM} \mathrm{N}$-acetylcysteine for $5 \mathrm{~min}$. For further details, see Methods.
PRP were incubated with various concentrations of nitroglycerin with or without $5.5 \mathrm{mM} N$-acetylcysteine for $5 \mathrm{~min}$ at $37^{\circ} \mathrm{C}$, after which the platelets were processed as described previously (14) and cGMP determined. Table III lists the values obtained and indicates that nitroglycerin increased resting cGMP from base-line levels fourfold without $N$-acetylcysteine (from $0.20 \pm 0.04$ to $0.80 \pm 0.04 \mathrm{pmol} / 10^{8}$ platelets) and up to 19 -fold with $N$-acetylcysteine (to $4.08 \pm 0.26 \mathrm{pmol} / 10^{8}$ platelets) at the highest nitroglycerin concentrations used $(390 \mu \mathrm{M})$.

The incubation time used in this experiment was chosen because inhibition by nitroglycerin of platelet aggregation in the presence of $\mathrm{N}$-acetylcysteine is maximal by $4 \mathrm{~min}$ and because subsequent experiments evaluating the time course of changes in platelet aggregation were carried out for as long as $5 \mathrm{~min}$ after addition of reactants (see below). As had been shown previously for nitroglycerin (14), no change in platelet cAMP from basal levels of $3.5 \pm 0.5 \mathrm{pmol} / 10^{8}$ platelets occurred in these experiments.

Time course of increase in platelet cGMP and inhibition of platelet aggregation. The temporal relationship between the increase in platelet CGMP and the decrease in ADP-induced platelet aggregation is shown in Fig. 2. Platelets in PRP were incubated at $37^{\circ} \mathrm{C}$ with $39 \mu \mathrm{M}$ nitroglycerin for $1 \mathrm{~min}$ and $5.5 \mathrm{mM} \mathrm{N}$-acetylcysteine was added at time zero. The incubation mixture was sampled at frequent times after addition of $\mathrm{N}$-acetylcysteine to determine the maximal rate of platelet aggregation in response to $11 \mu \mathrm{M}$ ADP, a value that typically was derived from no more than the first $15 \mathrm{~s}$ of the aggregation tracing after addition of ADP. (Please note: the extent of aggregation was not measured in these experiments because the time required to achieve the maximal extent of aggregation was prohibitively lengthy [up to $2 \mathrm{~min}$ ] relative to the time course of changes in cGMP that were being simultaneously measured.)

The concentration of nitroglycerin used $(39 \mu \mathrm{M})$ was chosen to permit measurement of aggregation rates that were less than completely inhibited, thereby permitting assessment of changes in rates at early times. Since the earliest time point sampled after addition of nitroglycerin was $1 \mathrm{~min}$, the large and transient increase in cGMP described by Mellion et al. (27) at very early times ( $<30 \mathrm{~s})$ after addition of nitroprusside or nitric oxide would not have been detected if it occurred in platelets exposed to nitroglycerin. Only the stable, late-appearing

Table III. Increase in Platelet cGMP by Nitroglycerin and Potentiation by $N$-Acetylcysteine*

\begin{tabular}{lll}
\hline & \multicolumn{2}{l}{ cGMP } \\
\cline { 2 - 3 } [Nitroglycerin] & $-N$-Acetylcysteine & $+N$-Acetylcysteine \\
\hline & pmol/108 platelets & \\
& $0.20 \pm 0.04$ & $0.22 \pm 0.04$ \\
3.9 & $0.26 \pm 0.02$ & $0.36 \pm 0.04$ \\
$3.9 \times 10^{-10}$ & $0.32 \pm 0.04$ & $0.53 \pm 0.02$ \\
$3.9 \times 10^{-6}$ & $0.54 \pm 0.08$ & $1.00 \pm 0.10$ \\
$3.9 \times 10^{-4}$ & $0.80 \pm 0.04$ & $4.08 \pm 0.26$
\end{tabular}

* Platelets in PRP were incubated for $5 \mathrm{~min}$ at $37^{\circ} \mathrm{C}$ with a range of nitroglycerin concentrations $\pm 5.5 \mathrm{mM} N$-acetylcysteine. Each point represents the mean $\pm S E M$ of three experiments done in duplicate. For further details, see Methods. 


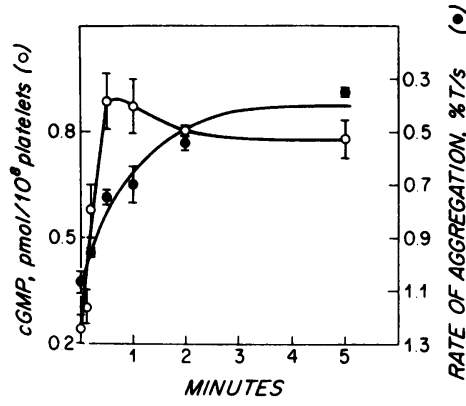

maximal rate of aggregation ( $\bullet$ ) (maximal change in percent transmittance per second) determined. Each point represents the mean \pm SEM of two or three experiments done in duplicate. Note that the ordinate is inverted for the rate of aggregation.

(i.e., beyond $1 \mathrm{~min}$ ) elevations of cGMP were measured in this experiment in order that they might be correlated better with changes in aggregation rate.

Fig. 2 shows that by $30 \mathrm{~s}$ after addition of $5.5 \mathrm{mM} \mathrm{N}$ acetylcysteine to platelets incubated with $39 \mu \mathrm{M}$ nitroglycerin, cGMP levels increased fourfold to $0.90 \mathrm{pmol} / 10^{8}$ platelets. Maximal inhibition of platelet aggregation rate (plotted with a reversed ordinate for purposes of comparison with the cGMP plot) lagged behind and was not attained until 2.5 min after addition of $\mathrm{N}$-acetylcysteine. Thus, this experiment demonstrates that the increase in platelet CGMP induced by nitroglycerin in the presence of $\mathrm{N}$-acetylcysteine preceded the attainment of maximal inhibition of platelet aggregation.

Effect of nitroglycerin on reduced glutathione concentration in platelets. Nitroglycerin undergoes denitrification in smooth muscle cells and hepatocytes through the action of an organic nitrate reductase (28). This denitrification requires GSH, the thiol undergoing oxidation in the process. GSH levels were measured in the quiescent platelet and found to be $4.0 \times 10^{-17}$ mol GSH/platelet, a value comparing favorably with published values (29). Incubating platelets with increasing concentrations of nitroglycerin led to a reduction in this intracellular GSH to $<10 \%$ of the base-line levels at $\sim 1 \mu \mathrm{M}$ nitroglycerin (Fig. 3).
Effect of $S$-nitroso- $N$-acetylcysteine on platelet aggregation. $S$-nitroso- $N$-acetylcysteine, synthesized from $N$-acetylcysteine and $\mathrm{NaNO}_{2}$ as described in Methods, markedly inhibited platelet aggregation in response to $11 \mu \mathrm{M}$ ADP, as depicted in Fig. 4. Incubating PRP for $5 \mathrm{~min}$ with $S$-nitroso- $N$-acetylcysteine inhibited aggregation with an $\mathrm{IC}_{50}$ of $6 \mathrm{nM}$; it is important to note that at equivalent concentrations of $N$-acetylcysteine or $\mathrm{NaNO}_{2}$ alone, no significant inhibition was noted (Fig. 1 and Table II), which supports the hypothesis that the adduct itself (and/or a metabolic product thereof) was the active species. Similar effects of $S$-nitroso- $N$-acetylcysteine were noted when using epinephrine $(12.5 \mu \mathrm{M})$ and calf skin collagen $(0.12 \mathrm{mg} /$ $\mathrm{ml}$ ) as agonists (Table IV).

Formation of S-nitroso-thiols by platelets on incubation with nitroglycerin. Platelets in PRP were incubated with $2.2 \mathrm{mM}$ nitroglycerin with or without $5.5 \mathrm{mM} \mathrm{N}$-acetylcysteine for $5 \mathrm{~min}$ at $37^{\circ} \mathrm{C}$ and extracted with TCA. The concentration of total nonprotein $S$-nitroso-thiol was determined by assaying free nitrite using diazotization and aromatic derivatization in the presence and absence of $\mathrm{HgCl}_{2}$ as described in Methods. Mercuric ions catalyze the hydrolysis of the nitroso moiety from the nitroso-thiol adducts and, in so doing, permit the determination of nitroso-thiols. In the absence of $\mathrm{N}$ acetylcysteine, $1.5 \pm 0.5 \mathrm{nmol} / 10^{8}$ platelets of $S$-nitroso-thiol was detected, while with $\mathrm{N}$-acetylcysteine, this value increased to $5.1 \pm 1.0 \mathrm{nmol} / 10^{8}$ platelets. No nitroso-thiol was detected in the absence of nitroglycerin. The effect of lesser concentrations of nitroglycerin was not assessed because sensitivity of the assay was limited and prohibitively large amounts of platelets would have been required. Protein-associated $S$-nitrosothiols were not measured in these experiments because only adducts to which the plasma membrane is permeable appear to be important for the effect of other organic nitrates noted in previous studies (27).

\section{Discussion}

These experiments demonstrate that the reduced thiol, $N$ acetylcysteine, markedly potentiates inhibition of platelet aggregation by nitroglycerin and other organic nitrate vasodilators.

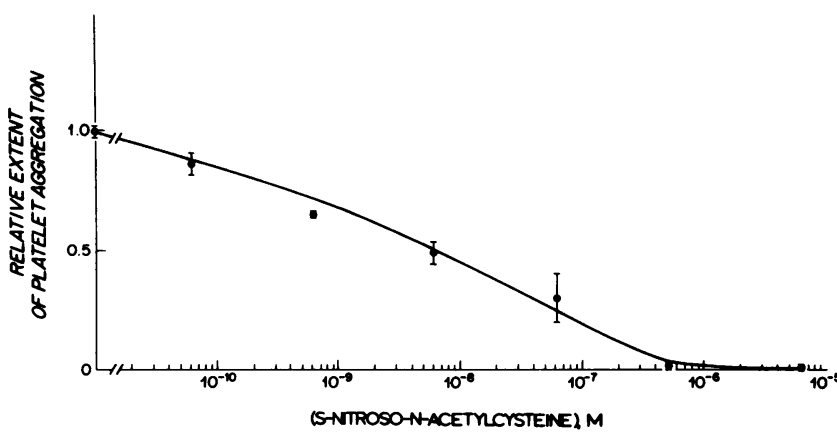

Figure 4. Effect of $S$-nitroso- $N$-acetylcysteine on ADP-induced platelet aggregation. Platelets in PRP were incubated for 5 min with increasing concentrations of $S$-nitroso- $N$-acetylcysteine at $37^{\circ} \mathrm{C}$, after which aggregation was induced by addition of $11 \mu \mathrm{M}$ ADP. Values on the ordinate reflect extent of aggregation relative to that in the absence of $S$-nitroso- $N$-acetylcysteine (corresponding to $85 \%$ maximal light transmittance). Each point represents the mean \pm SEM of three experiments done in duplicate. 
Table IV. Inhibition of Platelet Aggregation by $S$-Nitroso-N-Acetylcysteine*

\begin{tabular}{lc}
\hline Agonist & IC $_{\text {so }}$ \\
\hline & $\mu M$ \\
ADP $(11 \mu \mathrm{M})$ & $0.0061 \pm 0.0005$ \\
Epinephrine $(12.5 \mu \mathrm{M})$ & $0.100 \pm 0.001$ \\
Collagen $(0.12 \mathrm{mg} / \mathrm{ml})$ & $0.036 \pm 0.001$ \\
\hline
\end{tabular}

* Aggregation of platelets in PRP was induced by addition of agonist after incubating platelets at $37^{\circ} \mathrm{C}$ with various concentrations of $S$-nitroso- $N$-acetylcysteine for $5 \mathrm{~min}$. Each point represents the mean \pm SEM of two experiments done in duplicate. For further details, see Methods.

$\mathrm{N}$-Acetylcysteine was chosen for these experiments in place of other thiols because of its proved safety in humans and because of its demonstrated efficacy in potentiating the hypotensive effect of nitroglycerin in vivo (16). In smooth muscle cells, the relaxing effect of nitroglycerin was felt initially to be mediated by a nitroglycerin "receptor" (30), through which specific tolerance could be induced by prolonged exposure to nitroglycerin and reversed by reduced thiols (15). Further studies by Ignarro's group (31) suggested that the effect of nitroglycerin on smooth muscle cells was more complex than this and that CGMP was an essential mediator of nitroglycerin-induced vasodilatation. In these experiments, increases in smooth muscle cGMP in response to incubation with nitroglycerin preceded decreases in vascular tone, supporting the importance of the cyclic nucleotide.

In platelets, the inhibition of aggregation by nitroglycerin and other guanylate cyclase activators (nitroprusside, azide, and nitrite) suggests that cGMP is a crucial mediator of this inhibition (27). The fact that the maximal increase in platelet cGMP preceded the maximal inhibition of aggregation further supports this hypothesis. Controversy exists in the platelet literature about the role of CGMP in the aggregation response. Chiang and colleagues $(32,33)$, White et al. (34), and Glass et al. (35) demonstrated that platelet aggregation is associated with a rise in platelet cGMP levels, while the more recent studies of Claesson and Malmsten (36) and Weiss et al. (37) showed that cGMP either had no effect on platelet aggregation or else, at higher concentrations, inhibited aggregation. The data presented here show that, at least as far as inhibition of platelet aggregation by nitroglycerin is concerned, platelet cGMP levels maximally increase before maximal inhibition of aggregation, thereby arguing that elevation of cGMP is associated with inhibition of aggregation.

The ability of the reduced thiol, $N$-acetylcysteine, to potentiate the inhibitory effect of nitroglycerin further supports the importance of guanylate cyclase activity in the inhibition of platelet aggregation in these experiments. Preincubation with reduced thiol was essential for the expression of partially purified hepatic guanylate cyclase activity (38). In addition, the fact that hepatic and pulmonary (38) guanylate cyclase are rapidly inactivated by molecular oxygen, sulfhydryl oxidants, and thiol alkylating agents suggests that the redox state of sulfhydryl groups on guanylate cyclase determines its activation. Reduced thiols have also been found to stimulate activation of purified guanylate cyclase by nitroglycerin, among which are included cysteine, GSH, penicillamine, and dithiothreitol (26).

In the metabolism of nitroglycerin, denitrification occurs in a process requiring that GSH be catalyzed in liver by an organic nitrate reductase (30); the specific enzyme that serves this function is probably a glutathione- $S$-transferase (39). Denitrification is probably responsible not only for the metabolic fate of nitroglycerin, but also for its role as an activator of guanylate cyclase. Nitrite ion hydrolyzed from nitroglycerin reacts with reduced thiol to form $S$-nitroso-thiol compounds that, themselves, are very potent activators of guanylate cyclase (26), vasodilators (24), and, as shown both in this study for $S$ nitroso- $N$-acetylcysteine and in a recent study for other $S$ nitroso-thiols (40), extremely potent inhibitors of platelet activation. The loss of GSH and the appearance of $S$-nitrosothiols in platelets incubated with nitroglycerin supports the existence of this mechanism in platelets. The chemical identity of the $S$-nitroso-thiol(s) that form intracellularly has yet to be determined, but the possible candidates include $S$-nitrosoglutathione, $S$-nitroso-cysteine, or, perhaps, $S$-nitroso- $N$-acetylcysteine itself.

What $\mathrm{N}$-acetylcysteine does in this system is, as yet, not fully explained: it may serve as a source of thiol-reducing equivalents for the GSH system in the platelet; it may directly activate guanylate cyclase; it may serve as a reduced thiol source in the enzyme-catalyzed denitrification reaction; or it may serve as a substrate directly in the formation of $S$-nitrosothiols. Further studies are currently underway to clarify these issues.

In summary, the data presented here show the following: that $(a)$ nitroglycerin and other organic nitrates inhibit platelet aggregation and that this inhibition is potentiated markedly by the reduced thiol, $N$-acetylcysteine; that $(b)$ nitroglycerin, as other guanylate cyclase activators, inhibits platelet aggregation by inducing an increase in platelet cGMP levels that rise maximally before maximal inhibition of platelet aggregation; that (c) $S$-nitroso-thiols form when platelets are incubated with nitroglycerin; that $(d) S$-nitroso- $N$-acetylcysteine is an extremely potent inhibitor of platelet aggregation; and that $(e)$ such $S$ nitroso-thiols adducts are the active forms of nitroglycerin and other organic nitrates.

These data provide new insights into the possible effects of nitroglycerin on the vascular bed. At concentrations that are pharmacologically attainable in vivo, nitroglycerin reacts with GSH and/or other cellular thiols to form $S$-nitroso-thiols that not only vasodilate directly, but also markedly inhibit platelet aggregation. Inhibition of platelet activation and aggregation prevents synthesis of platelet-derived thromboxane $A_{2}$, and thereby further potentiates local direct vasodilating effects. In light of these data, the use of $S$-nitroso- $N$-acetylcysteine or other $S$-nitroso-thiols as antihypertensive or antiplatelet agents deserves further study.

\section{Acknowledgments}

The author wishes to express his appreciation to Drs. Andrew Schafer and Robert I. Handin for helpful discussions and to Ms. Jane Freedman for excellent technical support.

This work was supported by a Clinician-Scientist Award from the American Heart Association and, in part, by National Institutes of Health grants HL35014 and HL17513. 


\section{References}

1. Haerem, J. W. 1972. Platelet aggregates in intramyocardial vessels of patients dying suddenly and unexpectedly of coronary artery disease. Atherosclerosis. 15:199-213.

2. Haerem, J. W. 1974. Mural platelet microthrombi and major acute lesions of main epicardial arteries in sudden coronary death. Atherosclerosis. 19:529-541.

3. Ellis, E. F., O. Oelz, I. J. Roberts II, N. A. Payne, B. J. Sweetman, A. S. Nies, and J. A. Oates. 1976. Coronary arterial smooth muscle contraction by a substance released by platelets: evidence that it is thromboxane $A_{2}$. Science (Wash. DC). 193:1135-1137.

4. Greenwald, J. E., J. R. Bianchine, and L. K. Wong. 1979. The production of the arachidonate metabolite HETE in vascular tissue. Nature (Lond.). 281:588-589.

5. Needleman, P., P. S. Kulkarni, and A. Raz. 1977. Coronary tone modulation: formation and action of prostaglandins, endoperoxides, and thromboxanes. Science (Wash. DC). 195:409-411.

6. Marcus, A. J. 1978. The role of lipids in platelet function with particular reference to the arachidonic acid pathway. J. Lipid Res. 19: 793-801.

7. McGregor, M. 1983. Pathogenesis of angina pectoris and role of nitrates in relief of myocardial ischemia. Am. J. Med. 74(6B):21-27.

8. Warren, S. E., and G. S. Francis. 1978. Nitroglycerin and nitrate esters. Am. J. Med. 65:53-62.

9. Feldman, R. L., and C. R. Conti. 1981. Relief of myocardial ischemia with nitroglycerin: what is the mechanism? Circulation. 64: 1098-1102.

10. Frick, M. H., R. Balcon, D. Cross, and E. Sowton. 1968. Hemodynamic effects of nitroglycerin in patients with angina pectoris studied by the atrial pacing method. Circulation. 37:160-168.

11. Feldman, R. L., C. J. Pepine, and C. R. Conti. 1980. Magnitude of dilatation of large and small coronary arteries by nitroglycerin. Circulation. 64:324-333.

12. Hampton, J. R., M. J. G. Harrison, A. J. Honour, and J. R. A. Mitchell. 1967. Platelet behaviour and drugs used in cardiovascular disease. Cardiovasc. Res. 1:101-106.

13. Synek, P., K. Rysanek, H. Spankova, and M. Mlejnkova. 1970. The effect of ethanol and nitroglycerin on platelet aggregation. Activitas Nervosa Supplement. 12:77-82.

14. Schafer, A. I., R. W. Alexander, and R. I. Handin. 1980. Inhibition of platelet function by organic nitrate vasodilators. Blood. 55:649-654.

15. Needleman, P., B. Jakschik, and E. M. Johnson, Jr. 1973. Sulfhydryl requirement for relaxation of vascular smooth muscle. $J$. Pharmacol. Exp. Ther. 187:324-331.

16. Horowitz, J. D., E. M. Antman, B. H. Lorell, W. H. Barry, and T. W. Smith. 1983. Potentiation of the cardiovascular effects of nitroglycerin by $N$-acetylcysteine. Circulation. 68:1247-1253.

17. Born, G. V. R., and M. J. Cross. 1963. The aggregation of blood platelets. J. Physiol. (Lond.). 168:178-195.

18. Beutler, E., O. Duron, and B. M. Kelly. 1963. Improved method for the determination of blood glutathione. J. Lab. Clin. Med. 61:882-888.

19. Ellman, G. L. 1959. Tissue sulfhydryl groups. Arch. Biochem. Biophys. 82:70-77.

20. Habeeb, A. F. S. A. 1966. Chemical evaluation of conformational differences in native and chemically modified proteins. Biochim. Biophys. Acta. 115:440-454.

21. Habeeb, A. F. S. A. 1973. A sensitive method for localization of disulfide containing peptides in column effluents. Anal. Biochem. 56:310-311.

22. Snell, F. D., and C. T. Snell. 1949. Colorimetric Methods of Analysis. D. Van Nostrand Co., New York. Third ed. 804.

23. Saville, B. 1958. A scheme for the colorimetric determination of microgram amounts of thiols. Analyst. 83:670-672.
24. Ignarro, L. J., H. Lipton, J. C. Edwards, W. H. Baricos, A. L. Hyman, P. J. Kadowitz, and C. A. Gruetter. 1981. Mechanism of vascular smooth muscle relaxation by organic nitrates, nitrites, nitroprusside, and nitric oxide: evidence for the involvement of S-nitrosothiols as active intermediates. J. Pharmacol. Exp. Ther. 218:739-749.

25. Field, L., R. V. Dilts, R. Ravichandran, P. G. Lenhert, and G. E. Carnahan. 1978. An unusually stable thionitrite form $N$-acetylD,L-penicillamine: $x$-ray crystal and molecular structure of 2-(acetylamino)-2-carboxyl-1,1-dimethylethylthionitrite. J. Chem. Soc. (Perkin I):249-250.

26. Ignarro, L. J., and C. A. Gruetter. 1980. Requirement of thiols for activation of coronary artery guanylate cyclase gy glycerol trinitrate and sodium nitrite: possible involvement of S-nitrosothiols. Biochim. Biophys. Acta. 631:221-231.

27. Mellion, B. J., L. J. Ignarro, E. H. Ohlstein, E. G. Pontecorvo, A. L. Hyman, and P. J. Kadowitz. 1981. Evidence for the inhibitory role of guanosine 3',5'-monophosphate in ADP-induced human platelet aggregation in the presence of nitric oxide and related vasodilators. Blood. 57:946-955.

28. Needleman, P. 1976. Organic nitrate metabolism. Annu. Rev. Pharmacol. Toxicol. 16:81-93.

29. Akkerman, J. W. N. 1978. Regulation of carbohydrate metabolism in platelets. Thromb. Haemostasis. 39:712-724.

30. Needleman, P., and E. M. Johnson, Jr. 1973. Mechanism of tolerance development to organic nitrates. J. Pharmacol. Exp. Ther. 184:709-715.

31. Gruetter, C. A., D. Y. Gruetter, J. E. Lyon, P. J. Kadowitz, and L. J. Ignarro. 1981. Relationship between cyclic guanosine 3',5'monophosphate formation and relaxation of coronary arterial smooth muscle by glyceryl trinitrate, nitroprusside, nitrite, and nitric oxide: effects of methylene blue and methemoglobin. J. Pharmacol. Exp. Ther. 219:181-186.

32. Chiang, T. M., E. H. Beachey, and A. H. Kang. 1975. Interaction of chick skin collagen fragment ( $\alpha$-1-CB5) with human platelets. J. Biol. Chem. 250:6916-6922.

33. Chiang, T. M., S. N. Dixit, and A. H. Kang. 1976. Effect of cyclic $3^{\prime}, 5^{\prime}$-guanosine monophosphate on human platelet function. $J$. Lab. Clin. Med. 88:215-221.

34. White, J. G., N. D. Goldberg, R. D. Estensen, M. K. Haddox, and G. H. R. Rao. 1973. Rapid increase in platelet cyclic 3',5'guanosine monophosphate (CGMP) levels in association with irreversible aggregation, degranulation, and secretion. J. Clin. Invest. 52:89. (Abstr.)

35. Glass, D. B., J. M. Gerrard, D. Townsend, D. W. Carr, J. G. White, and N. D. Goldberg. 1977. The involvement of prostaglandin endoperoxide formation in the elevation of cyclic GMP levels during platelet aggregation. J. Cyclic Nucleotide Res. 3:37-44.

36. Claesson, H. E., and C. Malmsten. 1977. On the interrelationship of prostaglandin endoperoxide $G_{2}$ and cyclic nucleotides in platelet function. Eur. J. Biochem. 76:277-284.

37. Weiss, A., N. L. Baenziger, and J. P. Atkinson. 1978. Platelet release reaction and intracellular CGMP. Blood. 52:524-531.

38. Ignarro, L. J., P. J. Kadowitz, and W. H. Baricos. 1981. Evidence that regulation of hepatic guanylate cyclase activity involves interactions between catalytic site -SH groups and both substrate and activator. Arch. Biochem. Biophys. 208:75-86.

39. Habig, W. H., J. H. Keen, and W. B. Jakoby. 1975. GlutathioneS-transferase in the formation of cyanide from organic thiocyanates and as an organic nitrate reductase. Biochem. Biophys. Res. Commun. 64:501-506.

40. Mellion, B. J., L. J. Ignarro, C. B. Myers, E. H. Ohlstein, B. A. Ballot, A. L. Hyman, and P. J. Kadowitz. 1983. Inhibition of human platelet aggregation by S-nitroso-thiols: heme dependent activation of soluble guanylate cyclase and stimulation of cyclic GMP accumulation. Mol. Pharmacol. 23:653-664. 